\title{
Simulating Donnan equilibria based on the Nernst-Planck equation
}

\author{
Thomas Gimmi ${ }^{\mathrm{a}, \mathrm{b}, *}$, Peter Alt-Epping ${ }^{\mathrm{a}}$ \\ ${ }^{a}$ Rock-Water Interaction, Institute of Geological Sciences, University of Bern, CH-3012 Bern, \\ Switzerland \\ ${ }^{b}$ Laboratory for Waste Management, Nuclear Energy and Safety, Paul Scherrer Institut, CH-5132 \\ Villigen, Switzerland
}

\begin{abstract}
Understanding ion transport through clays and clay membranes is important for many geochemical and environmental applications. Ion transport is affected by electrostatic forces exerted by charged clay surfaces. Anions are partly excluded from pore water near these surfaces, whereas cations are enriched. Such effects can be modeled by the Donnan approach. Here we introduce a new, comparatively simple way to represent Donnan equilibria in transport simulations. We include charged surfaces as immobile ions in the balance equation and calculate coupled transport of all components, including the immobile charges, with the Nernst-Planck equation. This results in an additional diffusion potential that influences ion transport, leading to Donnan ion distributions while maintaining local charge balance. The validity of our new approach was demonstrated by comparing Nernst-Planck simulations using the reactive transport code Flotran with analytical solutions available for simple Donnan systems. Attention has to be paid to the numerical evaluation of the electrochemical migration term in the Nernst-Planck equation to obtain correct results for asymmetric electrolytes. Sensitivity simulations demonstrate the influence of various Donnan model parameters on simulated anion accessible porosities. It is furthermore shown that the salt diffusion coefficient in a Donnan pore depends on local concentrations, in contrast to the aqueous salt diffusion coefficient. Our approach can be easily implemented into other transport codes. It is versatile and facilitates, for instance, assessing the implications of different activity models for the Donnan porosity.
\end{abstract}

Keywords: Donnan model, clay, montmorillonite, ion transport, coupled transport, reactive transport, Nernst-Planck, electrochemical migration, anion exclusion, salt diffusion coefficient

\footnotetext{
${ }^{*}$ Corresponding author

Email addresses: thomas.gimmi@geo.unibe.ch, thomas.gimmi@psi.ch (Thomas Gimmi),
} alt-epping@geo.unibe.ch (Peter Alt-Epping) 


\section{Introduction}

Transport of ions through clays is important in many geochemical and environmental processes (Bourg et al., 2015; Neuzil \& Person, 2017). Clay behaves in many ways similar to a membrane because the clay surfaces are negatively charged. This affects the concentration of ions in the pore solution and their transport through the clay sample (Jougnot et al., 2009; Birgersson \& Karnland, 2009; Gimmi \& Kosakowski, 2011; Glaus et al., 2013; Tinnacher et al., 2016). The negative surface charge is compensated by cations near the surfaces. These cations are - more or less specifically — sorbed forming inner or outer sphere surface complexes or they occur as indifferently sorbing cations attracted only by Coulomb forces originating from the (net) surface charge (Delgado et al., 2005; Tournassat \& Steefel, 2015). The innermost layer of adsorbed cations, very close to the charged surface, is considered as Stern layer (Leroy \& Revil, 2004; Leroy et al., 2006). A diffuse layer (DL) develops adjacent to the Stern layer. There, anion and cation concentrations vary as a function of the distance to the surface, in response to electrostatic and thermal forces.

The ion distribution in the DL region, which depends on the "external" or bulk solution composition at infinite distance and the surface charge, can be modeled by the Poisson-Boltzmann equation, leading to the Gouy-Chapman model for simple geometries (Fig. 1). Alternatively, ion concentrations near charged surfaces can be calculated

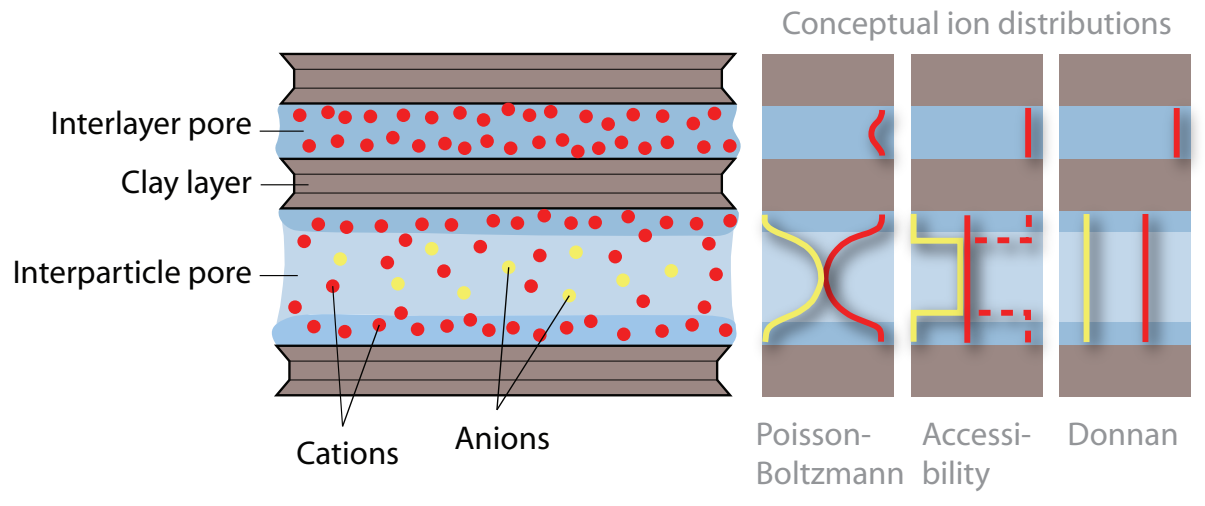

Figure 1: Schematic representation of anion and cation distributions near negatively charged clay surfaces (left) and of concepts to model such distributions (right). Blue areas represent pore water, with the darker blue illustrating schematically the Stern layer region. The solid lines (right) show schematically the anion (yellow) and cation (red) distributions in the pore water for the different concepts; the red dashed line illustrates the cation distribution including sorbed cations (which are not addressed in the accessibility concept).

using the Donnan approach (Donnan, 1911; Meyer \& Sievers, 1936), which is based on thermodynamic principles (Tournassat et al., 2016). The Donnan approach considers a uniform pore water composition in Donnan pores, i.e., pores affected by the surface charge (Fig. 1). For instance, it has been used to describe the average ion concentrations within the pore water of a clay sample (Birgersson \& Karnland, 2009) as a function of the bulk solution concentration with which the clay sample is in equilibrium. 
In a Donnan region cations are enriched and anions are depleted compared to the bulk solution. As a consequence, anion exclusion is typically observed when investigating the transport of anions through clays (Descostes et al., 2008; Van Loon et al., 2007; Tournassat \& Appelo, 2011; Wigger \& Van Loon, 2017). An accessible porosity smaller than the total, water-accessible porosity (more generally: a lower capacity) is assigned empirically to the transport of anions while assuming continuity of their concentration across the clay interface. This allows modeling fluxes of individual anion tracers through clay samples. However, in more complex situations, where concentrations of multiple anions and cations vary (such as in reactive transport), this empirical approach is no longer practical. A more general formulation is required that considers coupled anion and cation transport influenced by the bulk solution concentration.

So far, only the codes Phreeqc and CrunchFlow-MC offer this capability (Appelo \& Wersin, 2007; Steefel et al., 2015). Both codes follow a multi-porosity approach, where the pore space of a rock is divided into a charge-free (or free, or macro) porosity and a Donnan (or diffuse layer, or micro) porosity. Ion concentrations within the Donnan pore space in equilibrium with those in the charge-free pore space are calculated locally at every time step based on the Donnan equations. Implementing an explicit consideration of Donnan equilibrium into a reactive transport code requires major programming efforts. A different approach has been to couple other transport codes (e.g., Hydrus or generic Comsol $^{\mathrm{TM}}$ codes) to the chemical solver in Phreeqc or CrunchFlow-MC via an operator splitting approach (Wissmeier \& Barry, 2011; Nardi et al., 2014; Parkhurst \& Wissmeier, 2015; Muniruzzaman \& Rolle, 2016). However, without parallelization these coupled codes often lack computational performance.

Here we present an alternative, simpler way to represent Donnan equilibria: We consider charged clay surfaces as immobile anions in the pore solution and use the NernstPlanck (NP) equation to simulate coupled transport of all components. The immobile anions lead to a stationary diffusion potential relative to the bulk solution and to Donnan ion distributions maintaining local charge balance. We demonstrate the validity of our approach by comparing NP simulations using the reactive transport code Flotran (Lichtner, 2007) with analytical solutions of the Donnan equations for single symmetric and asymmetric electrolytes. We show that the 'standard' numerical evaluation of the electrochemical migration term in the NP equation produces errors for Donnan distributions with asymmetric electrolytes and propose a different evaluation of this term yielding correct results. Sensitivity calculations finally demonstrate the influence of various Donnan model parameters on simulated anion accessibilities. Our new approach can be easily implemented into other codes that consider multi-component diffusion based on the NP equation. It is versatile and allows for testing different assumptions regarding ion transport in the diffuse layer.

\section{Theory: Poisson-Boltzmann and Donnan equations}

The local ion distribution $C_{i}(x)$ in a diffuse layer adjacent to a charged surface can be described by the Boltzmann equation,

$$
C_{i}(x)=C_{i}^{B} \Gamma_{i}(x) \exp \left(\frac{-z_{i} F \varphi(x)}{R T}\right),
$$


where $C_{i}^{B}$ is the ion concentration in the bulk solution unaffected by the surface charge, $\Gamma_{i}(x)=\gamma_{i}^{B} / \gamma_{i}(x)$ the ratio of the activity coefficients in bulk solution and in the DL at position $x, z_{i}$ the charge number of the ion, $F$ the Faraday constant, $\varphi(x)$ the local electric potential (in $\mathrm{J} / \mathrm{C}=\mathrm{V}$ ), $R$ the universal gas constant, and $T$ the temperature. The Poisson equation,

$$
\nabla^{2} \varphi(x)=-\frac{F}{\varepsilon} \sum_{i} z_{i} C_{i}(x),
$$

links the electric potential to the local net charge density in the diffuse layer, with $\varepsilon$ being the dielectric permittivity of the medium. Combining Eqs. (1) and (2) leads to the Poisson-Boltzmann (PB) equation for a diffuse layer. Under certain conditions (e.g., in highly compacted clays) diffuse layers on different clay mineral surfaces may overlap. In this case the same equations apply but $C_{i}^{B}$ has to be replaced by the midplane concentration $C_{i}^{M}$, which can be related to $C_{i}^{B}$ and the width of the interlayer (Mitchell, 1993; Gonçalvès et al., 2007; Hedström \& Karnland, 2012). Solving the PB equation for a symmetric electrolyte and a single planar charged surface leads to the Gouy-Chapman (GC) model (Fig. 1). An innermost Stern layer of cations of finite size, over which the electric potential changes linearly, is considered in triple layer models. These innermost cations may be more rigidly bound forming for instance inner-sphere surface complexes (Bourg et al., 2017). The modified Gouy-Chapman model (MGC) (Tournassat et al., 2016) accounts for such a Stern layer by shifting the origin of the diffuse layer and adapting the net surface charge (or the potential) at the Stern/diffuse layer interface. The $\mathrm{PB}$ equations and the (M)GC model are mean field-approximations defined at the pore or molecular scale, and their solution for a clay sample would require precise knowledge of the pore structure (Tournassat et al., 2016). This information is not available for real clays.

The Donnan approach, in contrast, is well suited for the continuum scale used in most transport codes. It describes the thermodynamic effects of a charged surface on ion concentrations averaged over a representative pore volume. Equilibrating the chemical potentials of an external bulk solution $\left(\mu_{i}^{B}\right)$ with that of the internal solution within the Donnan pore space $\left(\mu_{i}^{D}\right)$ leads to

$$
\mu_{i}^{B, 0}+R T \ln a_{i}^{B}=\mu_{i}^{D, 0}+R T \ln a_{i}^{D}+z_{i} F \varphi_{m},
$$

where $a_{i}$ are activities, $\varphi_{m}$ is the Donnan potential and the superscripts $B, D$, and 0 refer to bulk solution, Donnan solution, and standard state, respectively. From Eq. (3), the ion concentration $C_{i}^{D}$ within the Donnan pore volume is

$$
C_{i}^{D}=C_{i}^{B} \Gamma_{i} \exp \left(-\frac{z_{i} F \varphi_{m}}{R T}\right)
$$

with $\Gamma_{i}=\gamma_{i}^{B} / \gamma_{i}^{D}$. The Donnan potential $\varphi_{m}$ depends on the surface charge and the solution composition. It is obtained from balancing the charge over the Donnan porosity through

$$
\sum_{i} z_{i} C_{i}^{D}=Q
$$

where $Q$ is the net surface charge per volume of Donnan solution (typically negative for clays). The net charge may be smaller (or larger) than the basic clay layer charge orig- 
inating from isomorphic substitution, in response to a partial shielding (or an addition) of charges by more strongly bound surface ions (e.g., a Stern layer) or protons.

The relative depletion or enrichment of ions within the Donnan space compared to the bulk solution,

$$
\xi_{i}=C_{i}^{D} / C_{i}^{B}
$$

depends on the ion charge according to Eq. (4). Comparing the concentration ratio $\xi$ for any two ions in a solution with charge $z_{i}$ and $z_{j}$, we have

$$
\left(\xi_{i} / \Gamma_{i}\right)^{1 / z_{i}}=\left(\xi_{j} / \Gamma_{j}\right)^{1 / z_{j}} .
$$

Thus, for ions with equal valence but opposite charge (e.g., $\mathrm{Na}^{+}, \mathrm{Cl}^{-}$) we have $\xi_{i}=$ $\Gamma_{i} \Gamma_{j} \xi_{j}^{-1}$, whereas for $\mathrm{Ca}^{2+}$ and $\mathrm{Cl}^{-}$we have $\xi_{\mathrm{Cl}}=\Gamma_{\mathrm{Cl}} \Gamma_{\mathrm{Ca}}^{1 / 2} \xi_{\mathrm{Ca}}^{-1 / 2}$, or for $\mathrm{Cl}^{-}$and $\mathrm{SO}_{4}^{2-}$ we have $\xi_{\mathrm{Cl}}=\Gamma_{\mathrm{Cl}} \Gamma_{\mathrm{SO}_{4}}^{-1 / 2} \xi_{\mathrm{SO}_{4}}^{1 / 2}$.

Combining Eqs. (4) and (5) leads to the general Donnan polynomial given by Sørensen \& Compañ (1996). Solutions for $C_{i}^{D}$ or $\xi_{i}$ for any polyelectrolyte can be derived from this polynomial by finding its real positive root. For an electrolyte with only two species $(i, j)$, the generic equation to be solved for $\xi_{i}$ is

$$
\xi_{i}^{\left(1-z_{j} / z_{i}\right)}-\frac{Q}{z_{i} C_{i}^{B}} \xi_{i}^{-z_{j} / z_{i}}-\Gamma_{i j}^{\left(1-z_{j} / z_{i}\right)}=0
$$

with $Q$ the net surface charge concentration and $\Gamma_{i j}$ the salt activity coefficient ratio,

$$
\Gamma_{i j}=\left(\Gamma_{i}^{z_{j}} \Gamma_{j}^{-z_{i}}\right)^{\frac{1}{z_{j}-z_{i}}} .
$$

A quadratic equation is obtained for any symmetric electrolyte $(1: 1,2: 2, \ldots)$ with the solution (Birgersson \& Karnland, 2009)

$$
\xi_{i}=\frac{z_{i}}{\left|z_{i}\right|} \frac{Q}{2 C_{i}^{B}}+\left(\left[\frac{Q}{2 C_{i}^{B}}\right]^{2}+\Gamma_{i} \Gamma_{j}\right)^{1 / 2} .
$$

Cubic equations are obtained for a 2:1 electrolyte (bivalent cation) such as $\mathrm{CaCl}_{2}$, which can be represented as

$$
\xi_{\mathrm{Cl}}^{3}+\frac{Q}{C_{\mathrm{Cl}}^{B}} \xi_{\mathrm{Cl}}^{2}-\Gamma_{\mathrm{Cl}}^{2} \Gamma_{\mathrm{Ca}}=0 \quad \text { and } \quad \xi_{\mathrm{Ca}}^{3 / 2}-\frac{Q}{2 C_{\mathrm{Ca}}^{B}} \xi_{\mathrm{Ca}}^{1 / 2}-\Gamma_{\mathrm{Cl}} \Gamma_{\mathrm{Ca}}^{1 / 2}=0
$$

Similarly, for a 1:2 electrolyte such as $\mathrm{Na}_{2} \mathrm{SO}_{4}$, we have

$$
\xi_{\mathrm{SO}_{4}}^{3 / 2}+\frac{Q}{2 C_{\mathrm{SO}_{4}}^{B}} \xi_{\mathrm{SO}_{4}}^{1 / 2}-\Gamma_{\mathrm{Na}} \Gamma_{\mathrm{SO}_{4}}^{1 / 2}=0 \text { and } \xi_{\mathrm{Na}}^{3}-\frac{Q}{C_{\mathrm{Na}}^{B}} \xi_{\mathrm{Na}}^{2}-\Gamma_{\mathrm{Na}}^{2} \Gamma_{\mathrm{SO}_{4}}=0
$$

Note that these equations all depend on the parameter $z_{i} C_{i}^{B} / Q$ or $C_{\text {eqv }}^{B} / Q$, i.e., the ratio of the bulk solution concentration $C_{\text {eqv }}^{B}$ of the anion or cation in (absolute) charge equivalents to the net surface charge concentration $Q$, and on the salt activity coefficient ratio $\Gamma_{i j}$ most often assumed to be 1 . An analytical solution for cubic equations is given, for instance, in Sørensen \& Compañ (1996), with which $\xi_{i}$ in Eqs. (11) and (12) can be obtained. 


\section{New approach to model Donnan equilibrium}

\subsection{General procedure}

In our new approach, instead of solving Eqs. (4) and (5) as in Phreeqc and CrunchFlowMC, we assign the net surface charge concentration $Q$ to immobile solutes of unit negative charge and include them in the balance volume (Fig. 2). Transport of ions is

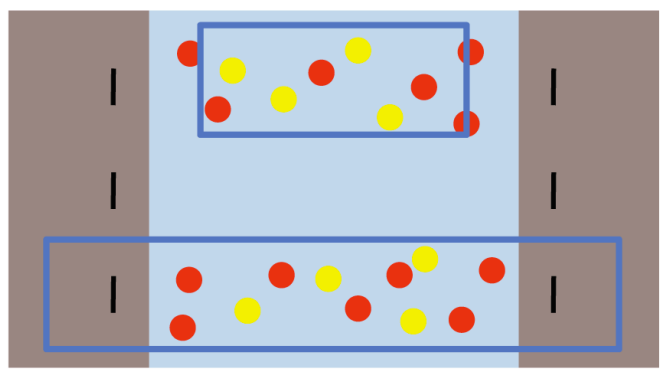

Figure 2: Illustration of conventional balance volume (top, only fluid phase) and balance volume used in our approach (bottom, fluid phase including surface charges).

then governed by the Nernst-Planck flux equation, which considers (ignoring advection here) ion diffusion according to the ion activity gradient and ion migration in an electrical field. In the absence of an external field and for quasi-static charges, the electrochemical migration term is related to the diffusion potential $\varphi$ that originates when ions diffuse at different rates (Cussler, 1984; Appelo \& Wersin, 2007; Steefel, 2008; Tournassat \& Steefel, 2015). We have in 1D (direction $x$ )

$$
\begin{aligned}
J_{i} & =-\theta D_{i}^{*} \frac{\partial C_{i}}{\partial x}-\theta D_{i} z_{i} C_{i} \frac{F}{R T} \frac{\partial \varphi}{\partial x} \\
& =-\theta D_{i}^{*} \frac{\partial C_{i}}{\partial x}+\theta D_{i} z_{i} C_{i} \frac{\sum_{j} z_{j} D_{j}^{*} \frac{\partial C_{j}}{\partial x}}{\sum_{j} z_{j}^{2} D_{j} C_{j}}
\end{aligned}
$$

where $J_{i}$ is the solute flux per total cross sectional area, $\theta$ the water content, $D_{i}$ the pore diffusion coefficient of component $i, D_{i}^{*}=D_{i}\left(1+\partial \ln \gamma_{i} / \partial \ln C_{i}\right)$ the pore diffusion coefficient including the activity term (sometimes called chemical diffusion coefficient), and $x$ the spatial coordinate. The activity gradients are most often neglected assuming $D_{i}^{*} \approx D_{i}$. The electrochemical migration term acts like a local advective flux for component $i$ that varies depending on its charge and on gradients, concentrations and mobilities of all other components. Thus, the electrochemical migration term either enhances or counteracts the local diffusive flux. When no electrical current occurs, the equilibrium is characterized by zero net ion flux with diffusive and electrochemical migration fluxes balancing each other. Concentration gradients of mobile ions may persist, if there is a heterogeneous distribution of immobile ions. At zero net flux,

$$
D_{i}^{*} \frac{\partial C_{i}}{\partial x}=D_{i} z_{i} C_{i} \frac{\sum_{j} z_{j} D_{j}^{*} \frac{\partial C_{j}}{\partial x}}{\sum_{j} z_{j}^{2} D_{j} C_{j}}
$$




$$
\frac{D_{i}^{*}}{z_{i} D_{i}} \frac{\partial \ln C_{i}}{\partial x}=\frac{\sum_{j} z_{j} D_{j}^{*} \frac{\partial C_{j}}{\partial x}}{\sum_{j} z_{j}^{2} D_{j} C_{j}} .
$$

The right-hand side of Eq. (15) represents the gradient of the diffusion potential $F /(R T) \partial \varphi / \partial x$ (see Eq. 13) and is identical for all components. Accordingly, for any two components $i$ and $j$ we can write

$$
\frac{D_{i}^{*}}{z_{i} D_{i}} \frac{\partial \ln C_{i}}{\partial x}=\frac{D_{j}^{*}}{z_{j} D_{j}} \frac{\partial \ln C_{j}}{\partial x}
$$

or, using the definition of $D_{i}^{*}$,

$$
\frac{1}{z_{i}}\left(\frac{\partial \ln C_{i}}{\partial x}+\frac{\partial \ln \gamma_{i}}{\partial x}\right)=\frac{1}{z_{j}}\left(\frac{\partial \ln C_{j}}{\partial x}+\frac{\partial \ln \gamma_{j}}{\partial x}\right) .
$$

Integrating this equation across an interface between a bulk solution (superscript $B$ ) and a solution affected by charged immobile ions (superscript $D$ ), we arrive at

$$
\frac{1}{z_{i}} \ln \left(\frac{\gamma_{i}^{D}}{\gamma_{i}^{B}} \frac{C_{i}^{D}}{C_{i}^{B}}\right)=\frac{1}{z_{j}} \ln \left(\frac{\gamma_{j}^{D}}{\gamma_{j}^{B}} \frac{C_{j}^{D}}{C_{j}^{B}}\right) .
$$

Substituting $\Gamma$ for $\gamma^{B} / \gamma^{D}$ and $\xi$ for $C^{D} / C^{B}$, we recover relation (7) characteristic for a Donnan equilibrium. Consequently, a diffusion potential created by immobile ions represents a Donnan potential, and Donnan concentration distributions can be calculated based on the NP equation.

\subsection{Implementation into reactive transport codes}

\subsubsection{Deriving average concentrations in the NP term}

The numerical implementation of the electrochemical migration term in the reactive transport code Flotran revealed that care has to be taken when local concentrations are assessed. Whereas the gradient terms in Eq. (13) can be directly calculated from concentration differences of two neighboring cells, some averaging is required to obtain the local concentrations $C$ of the electrochemical migration term in a discrete representation. Arithmetic averaging is typically used, but this leads to erroneous limiting anion distributions $\xi_{a} \rightarrow\left(z_{c}+z_{a}\right) /\left(z_{c}-z_{a}\right)$ (neglecting activity coefficients) for $C_{i}^{B} / Q \rightarrow 0$, i.e., dilute conditions, as shown in Appendix A and Fig. 3. Accordingly, erroneous limiting $\xi_{a}$ values of $1 / 3$ for $\mathrm{CaCl}_{2}$, of $1 / 2$ for a $3: 1$ electrolyte, or a tendency to $-1 / 3$ for a $1: 2$ electrolyte (bivalent anion) would be obtained.

Instead of an arithmetic average, a differential logarithmic average should be used to obtain local concentrations $\widetilde{C}_{k, k+1}$ at cell interfaces, defined as

$$
\widetilde{C}_{k, k+1}=\frac{d C}{d \ln C} \approx \frac{\Delta C}{\Delta \ln C}=\frac{C_{k+1}-C_{k}}{\ln C_{k+1}-\ln C_{k}}
$$

for the neighboring cells $k$ and $k+1$. When expanding Eq. (14) with local concentrations defined according to Eq. (19), correct limiting values of $\xi_{a} \rightarrow 0$ for $C_{i}^{B} / Q \rightarrow 0$ are obtained and results are consistent with Eq. (16). The differential logarithmic average leads to values between geometric and arithmetic mean. 


\subsubsection{Net surface charge concentration}

The surface charge concentration $Q$ per volume of clay pore water can be calculated as $C E C \rho_{b d} / \theta$, where $C E C$ is the cation exchange capacity (eq $/ \mathrm{kg}$ solid), $\rho_{b d}$ the bulk dry density, and $\theta$ the volumetric Donnan water content of the clay. This charge concentration $Q$ was then implemented via monovalent anions $\mathrm{X}^{-}$in the Donnan solution which exhibit a (quasi) zero diffusion coefficient.

A net charge lower than that given by the $C E C$ can also be attributed to the Donnan solution. Our approach treats $\mathrm{X}^{-}$as any other species in solution such that it can be involved in further reactions mimicking surface complexation or ion exchange reactions. In the latter case, the selectivities define the reaction constants, i.e., the $\log K$ entries in the thermodynamic database. However, in addition to selectivities a parameter quantifying the absolute interaction of a reference cation $\mathrm{R}$ with the exchanger is required (see Appendix B and Bradbury \& Baeyens 1998; Appelo et al. 2010). For instance, for a monovalent reference cation this parameter $K_{\mathrm{RX}}$ is defined as follows

$$
\mathrm{R}^{+}+\mathrm{X}^{-} \rightleftharpoons \mathrm{RX}, \quad K_{\mathrm{RX}}=\frac{(\mathrm{RX})}{(\mathrm{R})(\mathrm{X})},
$$

where ( ) denotes activity. The parameter $K_{\mathrm{Rx}}$ allows modeling limiting cases of full ion exchange $\left(K_{\mathrm{RX}} \gg 1\right.$, all surface charges compensated by exchangeable cations; ion selective) and full Donnan behavior $\left(K_{\mathrm{RX}} \ll 1\right.$, all surface charges compensated by ions in the Donnan space; charge selective), or of any intermediate situation. The net surface charge concentration $Q=C_{\mathrm{X}}$ is then variable depending on the specific composition of the bulk solution and all reaction constants and exchange selectivities. For symmetric electrolytes, the modification leads to a cubic instead of a quadratic equation for $\xi_{a}$ (cf. Eq. 10), as shown in Appendix C.

The approach can easily be generalized to multi-site sorption by distributing the total surface charge over different immobile surface ions $\mathrm{X}_{j}^{-}$. In this way, only certain sites may contribute to the Donnan equilibrium. Protons $\mathrm{H}^{+}$can be included in any such exchange reaction, or in additional sorption reactions, e.g.,

$$
\begin{aligned}
\mathrm{H}^{+}+\mathrm{X}_{j}^{-} & \rightleftharpoons \mathrm{HX}_{j}, \quad K_{\mathrm{HX}_{j}} \\
\mathrm{H}^{+}+\mathrm{HX}_{j} & \rightleftharpoons \mathrm{H}_{2} \mathrm{X}_{j}^{+}, \quad K_{\mathrm{H}_{2} \mathrm{X}_{j}^{+}} .
\end{aligned}
$$

The net Donnan charge then depends on the $\mathrm{pH}$.

Note that the net charge concentration $Q$ as well as, for heterovalent exchange, the $\log K_{A}^{R}$ depend on the water content $\theta$ (see also Appendix B). Accordingly, these parameters need to be updated when a system with time-dependent water contents should be modeled.

\subsection{Modeled systems}

In the following, we compare equilibrium ion distributions calculated numerically according to the NP equation (13) with analytical results of the Donnan distribution obtained from Eqs. (8) - (12). A 1D system including only two cells was modeled. One cell, with a fixed electrolyte concentration, represented the bulk solution, the other cell with a given $Q$ the Donnan porosity of a clay. In all simulations the activity coefficients were set to 1 . 
To demonstrate the effect of the averaging approach (i.e. arithmetic versus logaveraged), we also simulated transient in-diffusion of $\mathrm{Ca}^{2+}$ and $\mathrm{Cl}^{-}$into a $0.1 \mathrm{~m}$ thick heterogeneous clay sample, with a constant electrolyte solution at the inlet and a zero gradient boundary condition at the other end. The clay sample was heterogeneous with a smaller surface charge concentration $Q$ in the first $0.05 \mathrm{~m}$ and a larger concentration in the second $0.05 \mathrm{~m}$.

\section{Results}

\subsection{Verification of approach}

Equilibrium anion distributions $\xi_{\mathrm{Cl}}$ obtained numerically with immobile surface anions $\mathrm{X}^{-}$in the Donnan solution are presented in Fig. 3 as a function of the Donnan parameter $C_{e q v}^{B} / Q$ (i.e., the equivalent concentration of the bulk solution per surface charge concentration). For $\mathrm{NaCl}$ solutions, the numerical simulations (solid lines) match perfectly with the analytical results (crosses). At low concentration of the bulk solution,

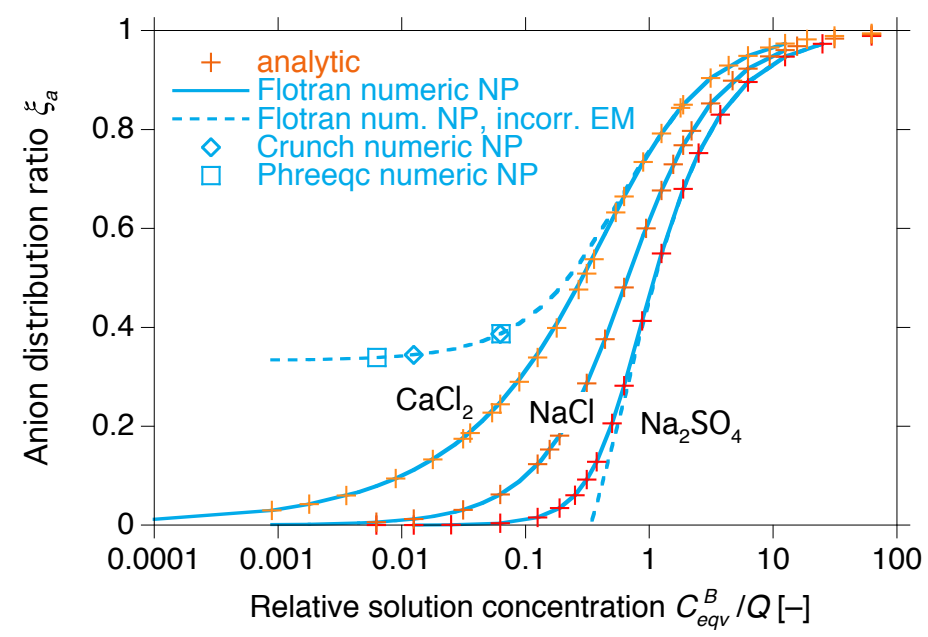

Figure 3: Donnan anion distribution ratios $\xi_{a}$ (anion accessibilities) as a function of the relative solution concentration $C_{e q v}^{B} / Q$ calculated based on the NP equation with Flotran (solid lines) compared to analytical results (crosses) for various electrolytes. Dashed lines: Flotran results using an arithmetic average when evaluating the electrochemical migration term; open squares and diamonds: NP solution with CrunchFlow and Phreeqc at three different values of $C_{e q v}^{B} / Q$.

$\mathrm{Cl}$ tends to be fully excluded from the Donnan solution, whereas the Donnan pore space becomes increasingly accessible to $\mathrm{Cl}$ at higher concentrations. For $\mathrm{CaCl}_{2}$ and $\mathrm{Na}_{2} \mathrm{SO}_{4}$ electrolytes, a perfect agreement was obtained if the differential logarithmic average in Eq. (19) was used. The anion exclusion (at given $C_{\text {eqv }}^{B} / Q$ ) is weaker in a $\mathrm{CaCl}_{2}$ electrolyte, and stronger in a $\mathrm{Na}_{2} \mathrm{SO}_{4}$ electrolyte, as compared to a $\mathrm{NaCl}$ electrolyte. For comparison, results using an arithmetic concentration average in the numerical evaluation of Eq. (13) are also shown (dashed lines in Fig. 3). In this case, $\xi_{\mathrm{Cl}}$ tended to $1 / 3$ for $\mathrm{CaCl}_{2}$ and to negative values for $\mathrm{Na}_{2} \mathrm{SO}_{4}$, instead of approaching zero at low 
bulk solution concentrations. The same incorrect result was obtained with Phreeqc and CrunchFlow using their NP implementation (diamonds and squares for $\mathrm{CaCl}_{2}$ in Fig. 3). Note that correct results were obtained with these two codes when their explicit Donnan implementation (i.e., the solution of Eqs. (4) and (5)) was used, which does not rely on the NP equation.

Anion distribution ratios $\xi_{a}$ for single electrolytes only were compared in Fig. 3. Results for a polyelectrolyte composed of $\mathrm{Na}_{2} \mathrm{SO}_{4}, \mathrm{NaCl}, \mathrm{CaCl}_{2}$ and $\mathrm{AlCl}_{3}$ obtained with the NP approach are presented as solid lines in Fig. 4. We see that $\xi_{\mathrm{SO}_{4}}$ and $\xi_{\mathrm{Cl}}$, i.e. the $\mathrm{SO}_{4}$ and $\mathrm{Cl}$ accessibility, increase compared to single $\mathrm{Na}$ electrolytes especially at low solution concentration. This effect results from stronger charge shielding by the bi- and trivalent cations. As in binary electrolytes, the bivalent $\mathrm{SO}_{4}$ is always more excluded than $\mathrm{Cl}$. The numerical results obtained with Flotran for $\xi_{\mathrm{Cl}}$ and $\xi_{\mathrm{SO}_{4}}$ are in perfect agreement with the analytical Eq. (7), i.e., we have $\xi_{\mathrm{SO}_{4}}=\xi_{\mathrm{Cl}}^{2}$.

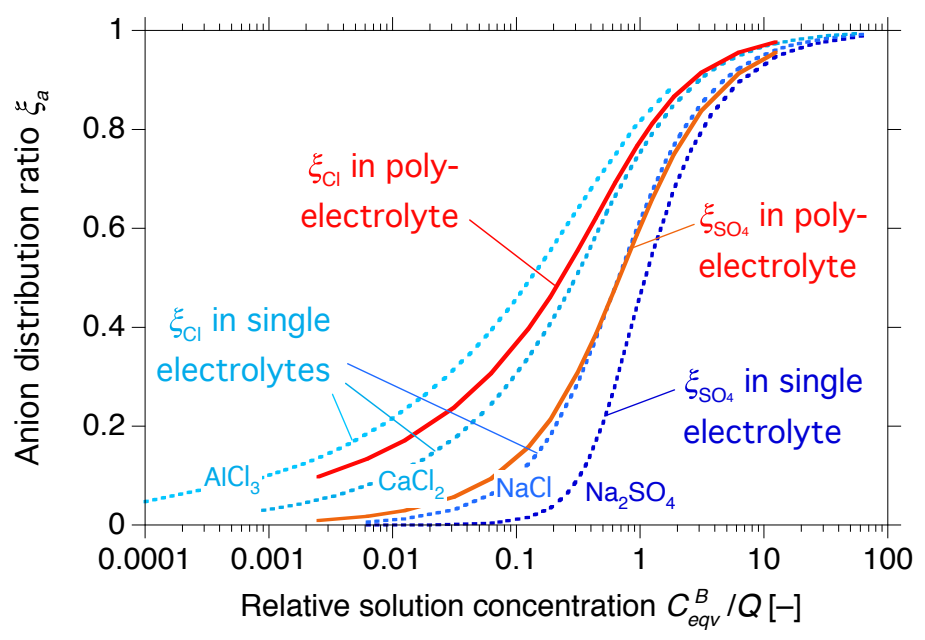

Figure 4: Anion distribution ratios $\xi_{\mathrm{Cl}}$ and $\xi_{\mathrm{SO}_{4}}$ for a polyelectrolyte (equal charge equivalents for $\mathrm{Na}$, $\mathrm{Ca}$, and $\mathrm{Al}$ and for $\mathrm{Cl}$ and $\mathrm{SO}_{4}$, solid lines), compared to those of single electrolytes (dotted lines). $C_{e q v}^{B}$ is the total charge equivalent in bulk solution.

\subsection{Sensitivity of anion accessibilities to Donnan model parameters}

A reduction of the net surface charge $Q$ due to a partial ion exchange behavior according to Eq. (20) leads to a slight steepening of the $\xi_{a}$ curve compared to a full Donnan model and to a gradual shift of the curve to lower $C_{\text {eqv }}^{B} / Q_{\mathrm{CEC}}$ (Fig. 5). Note that in this and the following figures, the $x$-axis is normalized by the constant $Q_{\mathrm{CEC}}=$ $C E C \rho_{b d} / \theta$ for the full Donnan case, calculated from the $C E C$ and the pore water content, and not by the variable $Q$. The slight steepening is related to a decrease of the net charge $Q=C_{\mathrm{X}}$ with higher solution concentration of the reference cation $\mathrm{R}$ (Na here) for a given $K_{\mathrm{RX}}$. The second observation, the gradual shift, depends on $K_{\mathrm{RX}}$. At low $K_{\mathrm{RX}}$, the full Donnan case is recovered with maximum anion exclusion. At high $K_{\mathrm{RX}}$, all surface charges are complexed already at low concentrations, leading in the limit to 
the full ion exchange case. For illustration purposes, $\mathrm{Cl}$ accessible pore fractions $(\mathrm{Cl}$ accessibilities, which are equivalent to $\xi_{C l}$ ) determined directly from the data of various field experiments in Opalinus Clay at the Mont Terri Rock Laboratory (Switzerland) are also shown as dots in Fig. 5.

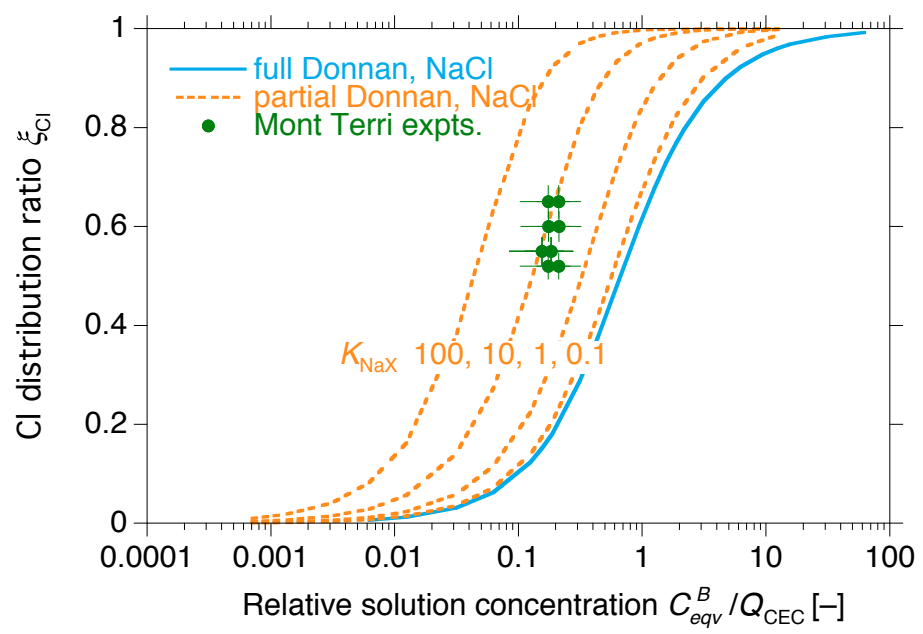

Figure 5: Effect of a decrease of the net surface charge $\mathrm{X}^{-}$due to ion exchange reactions according to Eq. (20) on the $\mathrm{Cl}$ distribution ratio, for increasing $K_{\mathrm{Nax}}$. Data (symbols) directly obtained from the DI-A1, DI-A2, DR, and DR-A field experiments in Opalinus Clay at the Mont Terri Rock Laboratory (Switzerland) are also shown (Van Loon et al., 2004; Wersin et al., 2008; Gimmi et al., 2014).

Anion accessibilities for bentonites measured in $\mathrm{NaCl}$ solutions of different ionic strengths by Muurinen et al. (1989) and Van Loon et al. (2007) and also shown by Birgersson \& Karnland (2009) are presented in Fig. 6. Here, they are shown as a function of the dimensionless Donnan parameter $C_{\text {eqv }}^{B} / Q_{\mathrm{CEC}}$, which allows a comparison of data for materials with different $C E C$, bulk dry density and water content. Two model curves are shown. The solid line represents the full Donnan case, where all pores are considered to belong to the Donnan space. The dashed line was obtained by assuming $5 \%$ charge-free porosity, i.e., by attributing only $95 \%$ of the porosity to the Donnan pore space, and by setting $K_{\mathrm{NaX}}=1$. The overall $\mathrm{Cl}$ accessibility shown in the figure is the weighted average of $\xi_{a}$ for the Donnan porosity and for the charge-free porosity. The data at higher bulk densities follow roughly the full Donnan model (Fig. 6). However, the full Donnan model underestimates the accessibility in samples at low bulk densities. A better match for the low-density data (mainly M89 $\rho_{b d} 1.2$, partly VL07 $\rho_{b d} 1.3$ ) is obtained by the modified model with $K_{\mathrm{NaX}}=1$ and $5 \%$ charge-free porosity. This increases the modeled $\mathrm{Cl}$ accessibility at all solution concentrations.

The calculations shown in Fig. 6 are based on the assumption that the Donnan and the charge-free pore volumes remain constant for all ionic strengths, i.e., for all bulk solution concentrations. However, this assumption may not be true as the Donnan porosity may change as a function of the ionic strength of the bulk solution, in particular when the bentonite shrinks or swells. A coupling between ionic strength and the Donnan volume 


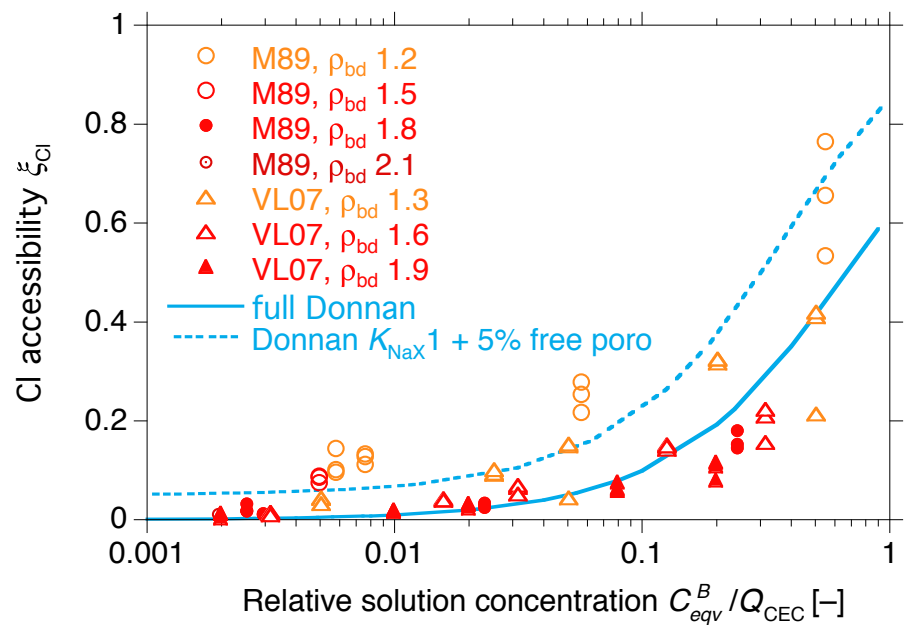

Figure 6: Data on $\mathrm{Cl}$ accessibilities $\xi_{\mathrm{Cl}}$ obtained from bentonites at various bulk dry densities (circles: Muurinen et al., 1989; triangles: Van Loon et al., 2007) compared to simulations for a full Donnan approach or a modified approach with $K_{\mathrm{NaX}}=1$ and $5 \%$ charge-free pores.

has been implemented into Phreeqc and CrunchFlowMC. In these codes the Donnan porosity $\theta_{D}$ can be calculated from the specific surface area $S$ per unit volume porous medium and the Debye length $\lambda_{D}$ (a characteristic length of a DL) multiplied by a scaling factor $a_{D}$ as

$$
\theta_{D}=a_{D} \lambda_{D} S
$$

This coupling is based on the idea that the DL thickness and thus the Debye length changes with ionic strength. The factor $a_{D}$ is an adjustable parameter that has to be estimated from experimental data. By linking the Donnan volume change to the Debye length, two approaches (Donnan and PB) are mixed that differ in their concepts and scale, but the approach may be useful to describe experimental data. Thus, for comparison purposes, Fig. 7 demonstrates the effects of variable Donnan and charge-free pore volumes on the modeled overall anion accessibility $\xi_{a}$ (again calculated as weighted average of $\xi_{a}$ for Donnan porosity and for charge-free porosity) for a $\mathrm{NaCl}$ solution. Type curves are shown for different values of the parameter $a_{D}$ (other parameters similar as for Opalinus Clay, i.e., $S=2.4 \cdot 10^{8} \mathrm{~m}^{2} \mathrm{~m}^{-3}$ or $S_{m}=100 \mathrm{~m}^{2} \mathrm{~g}^{-1}, \theta=0.15, C E C=0.1$ eq $\left.\mathrm{kg}^{-1}\right)$. The average anion accessibility for this model is generally larger than that calculated for the full Donnan case. Note that there is always a lower concentration limit for this model. It is reached when, for a given $a_{D}$, the Donnan porosity $\theta_{D}$ equals the total porosity $\theta$. For concentrations below this limit the Donnan porosity would exceed the total porosity. For large $a_{D}$, the limit is reached at relatively high $C_{e q v}^{B} / Q_{\mathrm{CEC}}$ values and the $\xi_{a}$ curve becomes similar to that assuming only Donnan pores, represented by the blue solid curve. For small $a_{D}$, the limit is reached at lower bulk solution concentrations. The smaller $a_{D}$, the smaller the fraction of the Donnan volume at a given $C_{\text {eqv }}^{B} / Q_{\mathrm{CEC}}$, and the larger the fraction of the charge-free volume. A smaller Donnan volume leads, for a given $C E C$, to a larger net $Q=C E C \rho_{b d} / \theta_{D}$ and thus to a comparably low anion 


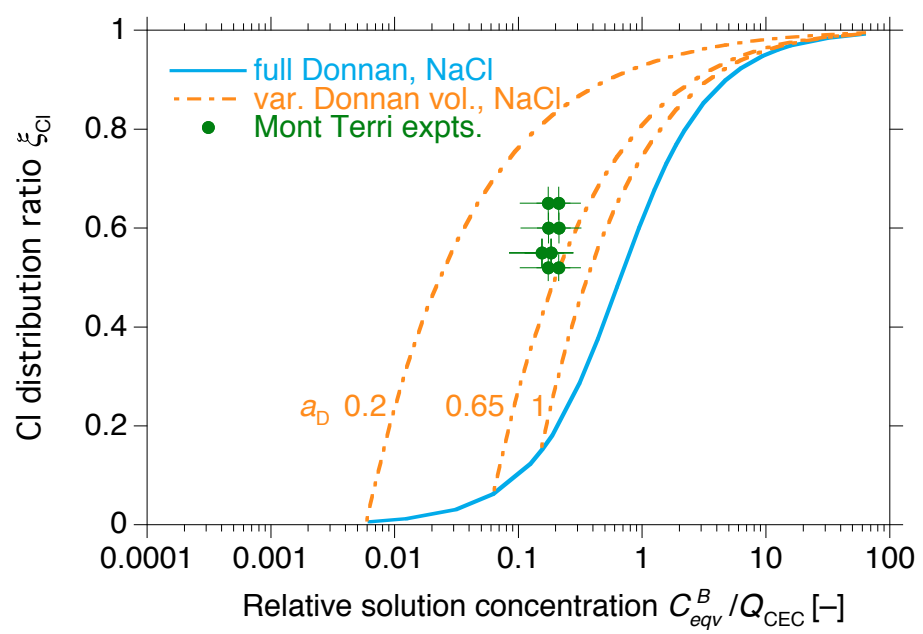

Figure 7: Effect of a variation of the Donnan volume with ionic strength on the Cl distribution ratio in a $\mathrm{NaCl}$ system for different multiplicators $a_{D}$ used to calculate the Donnan volume. Data (symbols) directly obtained from the DI-A1, DI-A2, DR, and DR-A field experiments in Opalinus Clay at the Mont Terri Rock Laboratory (Switzerland) are also shown (Van Loon et al., 2004; Wersin et al., 2008; Gimmi et al., 2014).

accessibility within the Donnan volume. But this effect is more than outweighed by the increase of the charge-free pore volume. Accordingly, the overall accessibility increases with decreasing $a_{D}$ at a given $C_{e q v}^{B} / Q_{\mathrm{CEC}}$. For comparison, the range of $\xi_{C l}$ determined directly from the data of field experiments in Opalinus Clay is again shown in Fig. 7.

\subsection{Evaluation of concentration averaging in the NP equation}

It was shown above that only the differential logarithmic average yields local concentrations that give correct Donnan distributions for asymmetric electrolytes. Given the potential implication of this result for the implementation of the Nernst-Planck equation into (reactive) transport codes, it is useful to look at the effect of different averaging techniques on simulation results in greater detail.

Fig. 8 shows concentration profiles for transient in-diffusion of $\mathrm{Ca}$ and $\mathrm{Cl}$ from a reservoir with a constant electrolyte concentration of $0.05 \mathrm{M} \mathrm{CaCl}_{2}$ into a $0.1 \mathrm{~m}$ thick heterogenous clay sample. The initial pore water has no $\mathrm{Cl}$ and there is a zero-gradient boundary condition at the opposite end of the sample. Tabulated aqueous diffusion coefficients $\left(2.032 \cdot 10^{-9} \mathrm{~m}^{2} \mathrm{~s}^{-1}\right.$ for $\mathrm{Cl}^{-}$and $0.792 \cdot 10^{-9} \mathrm{~m}^{2} \mathrm{~s}^{-1}$ for $\mathrm{Ca}^{2+}$, Flury \& Gimmi, 2002) were used, and sample parameters similar to those of Opalinus Clay (porosity 0.15, tortuosity factor 0.1). The distribution of the surface charge $Q$ is heterogeneous, with $0.8 \mathrm{M}$ between 0 and $0.05 \mathrm{~m}$ and $1.6 \mathrm{M}$ between 0.05 and $0.1 \mathrm{~m}$, respectively.

With the arithmetic average in the NP term, larger $\mathrm{Cl}$ concentrations in the pore water (and correspondingly larger Ca concentrations, not shown) are obtained compared to the logarithmic average. At $x=0.05 \mathrm{~m}$ a concentration jump occurs due to the increase in $Q$ leading to lower $\mathrm{Cl}$ concentrations between 0.05 and $0.1 \mathrm{~m}$. The anion accessibility 


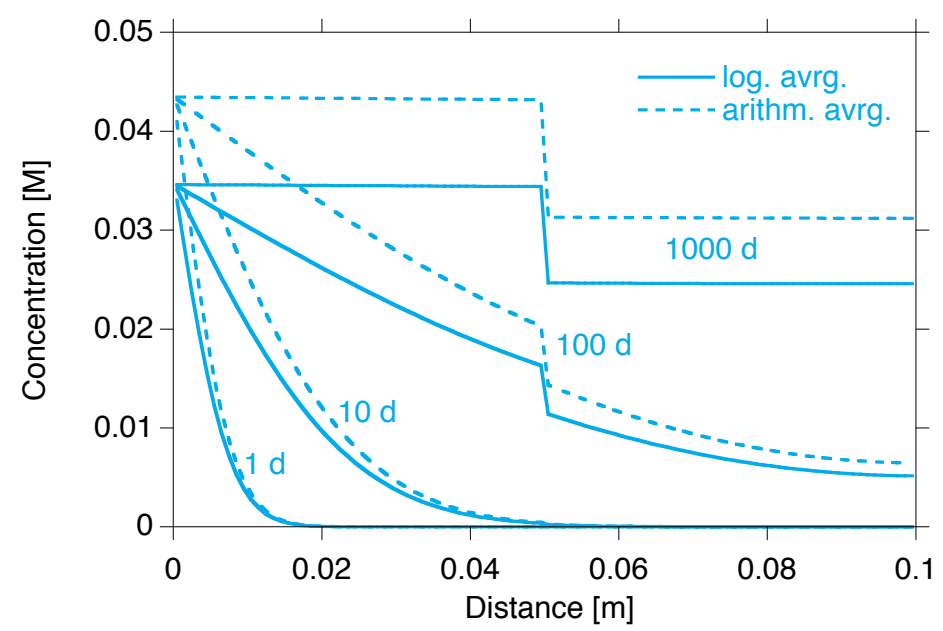

Figure 8: Results of in-diffusion of $\mathrm{Cl}^{-}$into a clay sample with $Q=0.8 \mathrm{M}(0-0.05 \mathrm{~m})$ and $Q=1.6 \mathrm{M}$ $(0.05-0.10 \mathrm{~m})$, calculated with our approach using the differential logarithmic average in the NP term (solid lines) or using the 'standard' arithmetic average. Left boundary: Constant $0.05 \mathrm{M} \mathrm{CaCl}_{2}$, right boundary: zero gradient. Sample porosity 0.15 , sample tortuosity factor 0.1 , water diffusion coefficient for $\mathrm{Cl}^{-} 2.032 \cdot 10^{-9} \mathrm{~m}^{2} \mathrm{~s}^{-1}$, for $\mathrm{Ca}^{2+} 0.792 \cdot 10^{-9} \mathrm{~m}^{2} \mathrm{~s}^{-1}$.

$\xi_{a}$ can be calculated from the equilibrium pore water concentration in the clay divided by the external $\mathrm{Cl}$ concentration $(0.1 \mathrm{M}$ ). It equals about 0.35 (left part, $Q=0.8 \mathrm{M}$ ) and 0.25 (right part, $Q=1.6 \mathrm{M}$ ) for the logarithmic average, which corresponds to the correct values as seen in Fig. 3 at the given $C_{e q v}^{B} / Q$ of 0.125 and 0.0625 , respectively. Using the arithmetic average these values are strongly overestimated $(0.44$ and 0.31$)$. In addition, only $\xi_{a}=0.44$ in the left part of the clay sample is consistent with the dashed line for the arithmetic average in Fig. 3, whereas the value in the right part is smaller than the value of 0.38 obtained from Fig. 3, which further points to an erroneous calculation of the fluxes for heterogeneous $Q$ with the arithmetic average.

Interestingly, using arithmetic averages in the NP term, as is the default in most (reactive) transport codes, does not affect multi-component simulations without immobile charges. Differences could be observed only in systems undergoing strongly transient behavior, but these differences remained negligible. The reason for this becomes clear when considering that for $Q \rightarrow 0$ or $C_{e q v}^{B} / Q \rightarrow \infty$, the equilibrium anion distribution ratio $\xi_{a}$ always equals 1 , and that no differences occur between the different averaging approaches at $\xi_{a}=1$ (see Fig. 3). Also, with $Q=0$, Eq. (A.6) derived from the arithmetic average correctly leads to $\xi_{a}=1$ for any finite bulk concentration. Nevertheless, to be more general in terms of model applicability, we recommend that the implementation of the electrochemical migration term into transport codes uses the differential logarithmic average of concentrations as described in Eq. (19). 


\section{Discussion}

\subsection{Cl accessibility in Opalinus Clay}

The $\mathrm{Cl}$ accessibilities in the field experiments shown in Figs. 5 and 7 are larger than those simulated for the full Donnan case and are better described, for instance, by a mixed ion exchange/Donnan model with $K_{\mathrm{NaX}} \approx 10$. One has to keep in mind, though, that (i) the composition of the pore solutions derived for Opalinus Clay are dominated by $\mathrm{Na}$ and $\mathrm{Cl}$, but other ions such as $\mathrm{Ca}, \mathrm{Mg}$, and $\mathrm{Sr}$ are also important, and that (ii) in addition to Donnan pores, charge-free pores and possibly inaccessible pores may also be present, requiring a multi-porosity model (Appelo \& Wersin, 2007; Tinnacher et al., 2016; Wigger \& Van Loon, 2017). Charge-free pores increase the limiting $\xi_{a}$ at low concentrations $\left(\xi_{a}>0\right)$, inaccessible pores decrease the limit at high concentrations $\left(\xi_{a}<1\right)$. Unfortunately, the limited range of the field data does not allow a direct estimation of these parameters. Furthermore, when comparing the same experimental data with a model with variable fractions of Donnan and charge-free pores in Fig. 7, it appears that such a model could describe the experimental data equally well when using a scaling factor $a_{D}$ around 0.5 . The limited range of $C_{\text {eqv }}^{B} / Q_{\mathrm{CEC}}$ for the field experiments in Opalinus Clay precludes any conclusion on whether a model with variable Donnan volume is better suited than one with constant volume. The recent DR-A field experiment at the Mont Terri Rock Laboratory, which is still under evaluation, was started to test such different modeling concepts.

\section{2. $\mathrm{Cl}$ accessibility in $\mathrm{Na}$ bentonite}

For the bentonite data in $\mathrm{NaCl}$ solutions shown in Fig. 6, a full Donnan model seems appropriate for the high bulk density data, but a mixed model with some charge-free pores and a partial complexation of the surface charge appears to be better suited for the data at lower bulk densities, or at least for the M89 $\rho_{b d} 1.2$ data. The samples at low density have a higher water content and probably also larger pore sizes. Accordingly, it could be plausible that a fraction of the pore water is not directly affected by any surface charge. But other factors, such as a dependency of the activity coefficient ratios (see Eq. 10) on the density of the samples, or the release of Ca from the non-smectite mineral fraction, could also play a role.

\subsection{Parameter problem for mixed ion exchange/Donnan models}

Considering a mixed model including both, ion exchange and Donnan distributions according to Eq. 20, raises some questions regarding the selectivities to be used. Published selectivities are usually derived considering ion exchange only. Thus it may be necessary to adapt the 'intrinsic' selectivities in a mixed model such that the overall apparent selectivities including also the cation excess in the Donnan pores match with literature data. Alternatively, published selectivities could be re-evaluated considering mixed ion exchange/Donnan models, provided the necessary parameters (notably the Donnan water content) are available. The potential effects depend on the water-rock ratio and the ionic strength of the solution in a batch experiment. 'Intrinsic' selectivities of two equivalent cations would tend to be more extreme than overall selectivities in a mixed system, if the Donnan distribution (assuming equal activity coefficients in bulk and Donnan solution) is only charge selective, but not ion selective. If, however, activity 
coefficient ratios vary between different ions, as recently discussed by Birgersson (2017), Donnan distributions also become ion selective and could possibly even fully account for reported selectivities between equally charged cations.

\subsection{Salt diffusion coefficient in Donnan pore water}

During transient diffusion of a binary electrolyte in Donnan pore water as in Fig. 8, both ions move in the end according to a single diffusion coefficient $D_{s}$ such that the charge is always balanced. In aqueous solutions, this salt (or electrolyte) diffusion coefficient can be calculated as a weighted harmonic mean of the individual ion diffusion coefficients $D_{a}$ and $D_{c}$ (e.g., Cussler, 1984),

$$
D_{s}=\frac{\left(z_{c}-z_{a}\right)}{\left(\frac{z_{c}}{D_{a}}-\frac{z_{a}}{D_{c}}\right)},
$$

with the weights given by the ion charges $z_{a}$ and $z_{c}$. In a Donnan pore space affected by surface charges, the salt diffusion coefficient is modified. Using the charge balance condition in the Donnan space, $z_{c} C_{c}+z_{a} C_{a}+z_{x} C_{x}=0$, with $C_{a}$ and $C_{c}$ the anion and cation concentrations in the Donnan pore space, respectively, $z_{x}$ the valence of the surface ion $\mathrm{X}$, and $C_{x}=Q$, we arrive at

$$
D_{s}=\frac{\left(z_{c}^{2} C_{c}+z_{a}^{2} C_{a}\right)}{\left(\frac{z_{c}^{2} C_{c}}{D_{a}}+\frac{z_{a}^{2} C_{a}}{D_{c}}\right)}=\frac{\left(z_{c}\left[1+\frac{z_{x} Q}{z_{a} C_{a}}\right]-z_{a}\right)}{\left(\frac{z_{c}}{D_{a}}\left[1+\frac{z_{x} Q}{z_{a} C_{a}}\right]-\frac{z_{a}}{D_{c}}\right)}=\frac{\left(z_{c}-z_{a}\left[1+\frac{z_{x} Q}{z_{c} C_{c}}\right]\right)}{\left(\frac{z_{c}}{D_{a}}-\frac{z_{a}}{D_{c}}\left[1+\frac{z_{x} Q}{z_{c} C_{c}}\right]\right)} .
$$

The salt diffusion coefficient in a Donnan pore is thus affected not only by the ion charges and ion diffusion coefficients, but also by the concentrations of the anion and the cation, or equally by the fraction of the surface charge per anion or cation charge. It is the ion with the lower (equivalent) concentration that tends to dominate the salt diffusion coefficient. This is typically the co-ion, that is, the anion if the surface is negatively charged and the cation if the surface is positively charged. If no surface charge is present $(Q=0)$, the aqueous salt diffusion coefficient is regained. In the limit of very large negative surface charge, $z_{x} Q /\left(z_{a} C_{a}\right) \rightarrow \infty$ (or equally $C_{a} \ll C_{c}$, or $z_{x} Q /\left(z_{c} C_{c}\right) \rightarrow-1$ because the counter-ion charge in absolute values has to be equal or larger than the surface charge) and $D_{s} \rightarrow D_{a}$. In the limit of very large positive surface charge, $z_{x} Q /\left(z_{c} C_{c}\right) \rightarrow \infty$ (or equally $C_{c} \ll C_{a}$, or $\left.z_{x} Q / z_{a} C_{a} \rightarrow-1\right)$ and $D_{s} \rightarrow D_{c}$.

In contrast to the aqueous salt diffusion coefficient, $D_{s}$ in a Donnan pore is not constant, but varies with time and position, because it depends on the local ion concentrations. For the example of $\mathrm{CaCl}_{2}$ in-diffusion in Fig. 8 , the variation is relatively small $\left(1.88 \cdot 10^{-9} \mathrm{~m}^{2} \mathrm{~s}^{-1} \leq D_{s} \leq 2.03 \cdot 10^{-9} \mathrm{~m}^{2} \mathrm{~s}^{-1}\right)$, as could be verified with our simulation results. In this case, the anion concentrations are always comparably low, and thus $D_{s}$ is always close to the limiting $D_{a}$. For larger maximum anion concentrations (larger external electrolyte concentration or smaller surface charge), $D_{s}$ will vary more. For instance, for the same boundary concentration of $0.05 \mathrm{M} \mathrm{CaCl}_{2}$ but a lower surface charge concentration of $Q=0.016 \mathrm{M}, D_{s}$ would vary between $D_{a}$ and $1.38 \cdot 10^{-9} \mathrm{~m}^{2} \mathrm{~s}^{-1}$, which is close to the lower limit of the aqueous $\mathrm{CaCl}_{2}$ salt diffusion coefficient $\left(1.335 \cdot 10^{-9} \mathrm{~m}^{2}\right.$ $\left.\mathrm{s}^{-1}\right)$. 


\section{Summary and conclusions}

We presented a new model for simulating Donnan equilibrium. It is based on immobile anions in the Donnan porosity that represent the surface charge. It can be easily implemented into codes that have the capability of solving the NP equation. Here we used an analytical approach to demonstrate the validity of the new method. Attention has to be paid to the numerical evaluation of the electrochemical migration term in the NP equation; a differential logarithmic average has to be used to calculate local concentrations, an arithmetic average leads to non-physical results. In sensitivity simulations we demonstrated that the anion accessibility may increase as a result of partial, ionic-strength dependent complexation of surface charges in a mixed model involving ion exchange and Donnan equilibrium. The accessibility also increases with increasing charge-free porosity.

It is possible with this approach to run transient 'dual continuum' simulations by treating Donnan and charge-free porosities as separate continua which are in diffusive exchange with each other. A second model dimension is then needed for a $1 \mathrm{D}$ problem (Jenni et al., 2017; Alt-Epping et al., 2018), or a third for a 2D problem. This on one hand limits the application of the approach, but on the other hand offers the possibility to investigate non-equilibrium effects, which cannot be done with an explicit implementation of Donnan equilibrium. Moreover, with this 'dual continuum' approach reactive transport processes can be evaluated in each porosity domain. It allows, for instance, to test the effect of activities on Donnan equilibrium, a topic that has reached more awareness recently (Birgersson, 2017). While the explicit Donnan implementation always assumes equal activity coefficients in bulk and Donnan solution, in our approach this is only one option. It is now possible to calculate activity coefficients explicitly for free and Donnan porosities (Alt-Epping et al., 2018), even with different activity models for each porosity domain. This allows one, for instance, to use an activity model that accounts for high ionic strengths in the Donnan porosity (either with or without the immobile ions $\mathrm{X}^{-}$). Note that inclusion of the activity gradients in the electrochemical migration term in Eq. (13) is then required.

Finally, transient simulations in heterogeneous systems can be easily performed as shown for instance in Fig. 8. In more complex cases, where the porosity and/or the surface charge changes with time, an update of the concentration $Q$ is required (similarly as for other solutes). Also, if advection is considered in the Donnan space (being meaningful or not), the velocity of $\mathrm{X}$ needs to be set to zero, which may require additional code modifications. Transient simulations allowed to check the analytical relation for the salt diffusion coefficient of a binary electrolyte in Donnan pores. This Donnan salt diffusion coefficient is time- and position dependent, in contrast to the salt diffusion coefficient in aqueous solution, and can vary between the latter and the co-ion diffusion coefficient. Overall, the presented approach to simulate Donnan systems is simple in many cases, flexible, and has a large potential for further applications.

\section{Acknowledgement}

Partial financial support by Nagra, the Swiss Cooperative for the Disposal of Radioactive Waste, is acknowledged. 


\section{Appendix A}

When numerically evaluating the electrochemical migration flux term in the Nernst Planck equation (Eq. (13) in the main manuscript) in a discretized representation, some averaging is required to obtain the local concentrations $C_{j}$. In the following, we show that using an arithmetic average, which appears to be the standard implementation in numerical codes, leads to erroneous limiting values for steady-state Donnan ion distributions and thus generally to erroneous electrochemical migration fluxes in Donnan systems.

We start with the steady-state version of the Nernst-Planck flux equation as given in Eq. (14) in the main manuscript. For a single electrolyte $\mathrm{C}_{\left|z_{a}\right|} \mathrm{A}_{z_{c}}$ consisting of cation $\mathrm{C}$ and anion $\mathrm{A}$ with valences $z_{c}$ and $z_{a}$, respectively, the discretized form of this equation leads to

$$
\frac{D_{a}^{*}}{D_{a}} \frac{1}{z_{a}} \frac{\Delta C_{a}}{C_{a}}=\frac{D_{c}^{*}}{D_{c}} \frac{1}{z_{c}} \frac{\Delta C_{c}}{C_{c}} .
$$

where $C$ is the concentration, $D$ the pore diffusion coefficient, $D^{*}=D(1+d \ln \gamma / d \ln C)$ the pore diffusion coefficient including the activity term (sometimes denoted as chemical diffusion coefficient), and subscripts $a$ and $c$ refer to the anion and the cation, respectively.

We consider a model with two neighboring cells at equilibrium, one representing the bulk solution (superscript $B$ ) and the other the Donnan solution in a clay (superscript $D)$. The concentration differences can then be given as

$$
\Delta C_{a}=C_{a}^{D}-C_{a}^{B} \quad \text { and } \quad \Delta C_{c}=C_{c}^{D}-C_{c}^{B} .
$$

Now, when using arithmetic averages for $C_{a}$ and $C_{c}$ in Eq. (A.1) and setting $r_{a}=D_{a}^{*} / D_{a}$ and $r_{c}=D_{c}^{*} / D_{c}$, we have

$$
\frac{r_{a}}{z_{a}} \frac{C_{a}^{D}-C_{a}^{B}}{C_{a}^{D}+C_{a}^{B}}=\frac{r_{c}}{z_{c}} \frac{C_{c}^{D}-C_{c}^{B}}{C_{c}^{D}+C_{c}^{B}} .
$$

We then make use of the electroneutrality conditions in the bulk and the Donnan solution,

$$
C_{c}^{B}=-\frac{z_{a}}{z_{c}} C_{a}^{B} \quad \text { and } \quad C_{c}^{D}=-\frac{z_{a}}{z_{c}} C_{a}^{D}-\frac{z_{x}}{z_{c}} Q,
$$

where $Q$ is the net surface charge concentration and $z_{x}$ is the valence of the surface ion $\mathrm{X}$ taken as -1 , to eliminate the cation concentrations in Eq. (A.3), and use the anion concentration distribution ratio (or anion exclusion parameter) $\xi_{a}$,

$$
\xi_{a}=C_{a}^{D} / C_{a}^{B}
$$

This leads finally to

$$
\begin{aligned}
& \left(r_{c} z_{a}-r_{a} z_{c}\right) \xi_{a}^{2}+\left(r_{c} z_{a}-r_{a} z_{c}\right) \frac{z_{x}}{z_{a}} \frac{Q}{C_{a}^{B}} \xi_{a}+ \\
& \left(r_{c} z_{a}+r_{a} z_{c}\right) \frac{z_{x}}{z_{a}} \frac{Q}{C_{a}^{B}}-\left(r_{c} z_{a}-r_{a} z_{c}\right)=0 .
\end{aligned}
$$

In the limit of $C_{a}^{B} \rightarrow 0$ and thus $\xi_{a} \ll 1$, the first term on the left hand side becomes much smaller than the second term, and the last term on the left hand side much smaller than the second to last term. Accordingly, for $C_{a}^{B} \rightarrow 0$ we arrive at

$$
\left(r_{c} z_{a}-r_{a} z_{c}\right) \frac{z_{x}}{z_{a}} \frac{Q}{C_{a}^{B}} \xi_{a}+\left(r_{c} z_{a}+r_{a} z_{c}\right) \frac{z_{x}}{z_{a}} \frac{Q}{C_{a}^{B}}=0
$$




$$
\xi_{a}=\frac{r_{c} z_{a}+r_{a} z_{c}}{r_{a} z_{c}-r_{c} z_{a}}
$$

For $r_{a} / r_{c}=1$, we thus have

$$
\xi_{a}=\frac{z_{a}+z_{c}}{z_{c}-z_{a}}
$$

as was observed in numerical simulations based on the arithmetic average. According to this relation, $\xi_{a}$ could even become negative when $\left|z_{a}\right|>z_{c}$, which is physically impossible. This demonstrates that discretized electrochemical migration fluxes in Donnan systems with immobile charges are incorrect when using an arithmetic average to calculate this term, both in transient and steady situations. In contrast, the limit of $\xi_{a}$ for $C_{a}^{B} \rightarrow 0$ is well behaving and approaches the correct result when using the differential logarithmic average proposed in Eq. (19) of the main manuscript, as can be verified in the same manner. Hence the proposed averaging leads to correct electrochemical migration fluxes in all cases.

\section{Appendix B}

Here we outline the conversion of selectivities to reaction constants as needed for mixed ion exchange/Donnan systems in the present approach. A mixed ion exchange/Donnan distribution model can be simulated by considering immobile anions $\mathrm{X}^{-}$in the Donnan solution representing surface charges and a set of reactions of cations A with valence $z_{A}$ with these immobile species $\mathrm{X}$ according to

$$
\mathrm{A}+z_{A} \mathrm{X} \rightleftharpoons \mathrm{AX}_{z_{A}}, \quad K_{\mathrm{AX}}=\frac{\left(\mathrm{AX}_{z_{A}}\right)}{(\mathrm{A})(\mathrm{X})^{z_{A}}},
$$

where $K_{\mathrm{Ax}}$ is the formation constant and ( ) denotes activity. Two such reactions describe an exchange reaction between cation $\mathrm{A}$ and the reference cation $\mathrm{R}$ with valence $z_{R}$ (e.g., Appelo \& Postma, 2005),

$$
z_{R} \mathrm{~A}+z_{A} \mathrm{RX}_{z_{R}} \rightleftharpoons z_{A} \mathrm{R}+z_{R} \mathrm{AX}_{z_{A}}
$$

The exchange constant $K_{\mathrm{R}}^{\mathrm{A}}$ for this reaction is defined as

$$
K_{\mathrm{R}}^{\mathrm{A}}=\frac{K_{\mathrm{AX}}^{z_{R}}}{K_{\mathrm{RX}}^{z_{A}}}=\frac{(\mathrm{R})^{z_{A}}\left(\mathrm{AX}_{z_{A}}\right)^{z_{R}}}{(\mathrm{~A})^{z_{R}}\left(\mathrm{RX}_{z_{R}}\right)^{z_{A}}}
$$

Selectivities ${ }^{c} K_{\mathrm{R}}^{\mathrm{A}}$ for ion exchange reactions using the Gaines-Thomas convention are defined as

$$
{ }^{c} K_{\mathrm{R}}^{\mathrm{A}}=\frac{(\mathrm{R})^{z_{A}} N_{\mathrm{A}}^{z_{R}}}{(\mathrm{~A})^{z_{R}} N_{\mathrm{R}}^{z_{A}}},
$$

where $N_{i}$ denotes equivalent fraction of $i$ defined as charge equivalents occupied by cation $i$ on the exchanger per total equivalents of the exchanger.

According to these definitions, exchange constants $K_{\mathrm{R}}^{\mathrm{A}}$ are related to exchange selectivities ${ }^{c} K_{\mathrm{R}}^{\mathrm{A}}$ (see Appendix A of Bradbury \& Baeyens 1998) as

$$
K_{\mathrm{R}}^{\mathrm{A}}={ }^{c} K_{\mathrm{R}}^{\mathrm{A}} \frac{z_{R}}{z_{A}}\left[\frac{\theta C_{0}}{\rho_{b d} C E C}\right]^{z_{A}-z_{R}},
$$


where $\theta\left[\mathrm{L} \mathrm{L}^{-1}\right]$ is the volumetric water content, $\rho_{b d}\left[\mathrm{~kg} \mathrm{~L}^{-1}\right]$ the bulk dry density, and $C E C\left[\mathrm{~mol} \mathrm{~kg}^{-1}\right]$ the cation exchange capacity of the material, and $C_{0}$ (typically $1 \mathrm{M}$ ) the standard state concentration for solutes (consistent units have to be used within the square brackets). Note that the $C E C$ can be considered as the 'standard state' value for equivalent fractions, so the term $C_{0} / C E C$ is a ratio of standard state values. Also, the term in square brackets is only relevant for heterovalent exchange and disappears if ions of equal valence exchange. In logarithmic form, the relation is

$$
\log K_{\mathrm{R}}^{\mathrm{A}}=\log { }^{c} K_{\mathrm{R}}^{\mathrm{A}}+\log \frac{z_{R}}{z_{A}}+\left(z_{A}-z_{R}\right) \log \left[\frac{\theta C_{0}}{\rho_{b d} C E C}\right] .
$$

We see from this equation that for heterovalent exchange the exchange constants depend on the bulk dry density and the porosity of the material and thus may have to be updated during transient simulations (assuming that the selectivities remain constant), as mentioned in Bradbury \& Baeyens (1998).

Having obtained the exchange constants $K_{\mathrm{R}}^{\mathrm{A}}$ from the selectivities of each cation, all but one reaction constants $\mathrm{K}_{A X}$ of Eq. (B.1) are defined; they can be calculated from Eq. (B.3) as

$$
K_{\mathrm{AX}}=K_{\mathrm{R}}^{\mathrm{A}^{1 / z_{R}}} K_{\mathrm{RX}^{z_{A} / z_{R}}}
$$

One reaction constant, e.g., $K_{\mathrm{Rx}}$ for the interaction of the reference cation with the immobile anion, remains a free parameter (see Eq. (20) of the main manuscript). Depending on the value of $K_{\mathrm{RX}}$, the system may be dominated by ion exchange or by Donnan reactions, or show an intermediate behavior.

\section{Appendix C}

A cubic equation for the anion accessibility $\xi_{a}$ is derived here for a symmetric electrolyte (e.g., $\mathrm{NaCl}$ ) in a mixed complexation/Donnan system. This equation can be used to verify numerical solutions for such a mixed system. Ion exchange reactions lead to a decrease of the net surface charge concentration $Q$ in response to the partial occupation of the exchanger. Starting with Eq. (8) of the main manuscript, we can derive a modified equation for $\xi_{a}$, the anion distribution ratio, for a symmetric electrolyte. The net surface charge $Q$ equals the concentration $C_{\mathrm{X}}$ of the uncomplexed immobile anions $\mathrm{X}^{-}$. It can be given as

$$
C_{\mathrm{X}}=Q_{\mathrm{CEC}}-C_{\mathrm{NaX}},
$$

where $Q_{\mathrm{CEC}}$ is the maximum surface charge concentration given by the $C E C$ and $C_{\mathrm{NaX}}$ is the concentration of the $\mathrm{NaX}$ exchange complexes. This latter can be obtained from Eq. (B.1) or Eq. (20) of the main manuscript as

$$
C_{\mathrm{NaX}}=C_{\mathrm{X}} C_{\mathrm{Na}}^{D} \Gamma_{\mathrm{Na}, \mathrm{X}} K_{\mathrm{NaX}},
$$

where $\Gamma_{\mathrm{Na}, \mathrm{X}}=\gamma_{\mathrm{X}} \gamma_{\mathrm{Na}}^{D} / \gamma_{\mathrm{NaX}}$ is a term combining all activity coefficients. Combining the last two equations and setting $K_{\mathrm{NaX}}^{\prime}=\Gamma_{\mathrm{Na}, \mathrm{X}} K_{\mathrm{NaX}}$, the net surface charge concentration can be calculated as

$$
Q=C_{\mathrm{X}}=\frac{Q_{\mathrm{CEC}}}{1+C_{\mathrm{Na}}^{D} K_{\mathrm{NaX}}^{\prime}} .
$$


We see already from this equation that the net surface charge $Q$ will tend to $Q_{\mathrm{CEC}}$ for very small $K_{\mathrm{NaX}}^{\prime}$, and to zero for very large $K_{\mathrm{Nax}}^{\prime}$. The concentration $C_{\mathrm{Na}}^{D}$ is unknown a priori, but we can eliminate it by making use of the relations $\xi_{\mathrm{Na}}=C_{\mathrm{Na}}^{D} / C_{\mathrm{Na}}^{B}, \xi_{\mathrm{Na}}=1 / \xi_{\mathrm{Cl}}$, and $C_{\mathrm{Na}}^{B}=C_{\mathrm{Cl}}^{B}$. This leads to

$$
Q=C_{\mathrm{X}}=\frac{Q_{\mathrm{CEC}}}{1+\frac{C_{\mathrm{Na}}^{B}}{\xi_{\mathrm{Cl}}} K_{\mathrm{NaX}}^{\prime}} .
$$

Plugging this expression into Eq. (8) of the main manuscript expressed for $\mathrm{NaCl}$, we arrive finally at a cubic equation that describes the anion concentration distribution ratio $\xi_{\mathrm{Cl}}$ for a $\mathrm{NaCl}$ electrolyte in a mixed complexation/Donnan system:

$$
\xi_{\mathrm{Cl}}^{3}+\left(\frac{Q_{\mathrm{CEC}}}{C_{\mathrm{Cl}}^{B}}+C_{\mathrm{Cl}}^{B} K_{\mathrm{Nax}}^{\prime}\right) \xi_{\mathrm{Cl}}^{2}-\Gamma_{\mathrm{NaCl}}^{2} \xi_{\mathrm{Cl}}-\Gamma_{\mathrm{NaCl}}^{2} C_{\mathrm{Cl}}^{B} K_{\mathrm{NaX}}^{\prime}=0
$$

The numerical simulations obtained for $\mathrm{NaCl}$ in such a mixed system were verified by solutions of the above cubic equation.

\section{References}

Alt-Epping, P., Gimmi, T., Wersin, P., \& Jenni, A. (2018). Incorporating electrical double layers into reactive-transport simulations of processes in clays by using the Nernst-Planck equation: A benchmark revisited. Applied Geochemistry, 89, 1-10 doi:https://doi.org/10.1016/j.gca.2018.04.003https://doi.org/10.1016/j.apgeochem.2017.10.018.

Appelo, C. A. J., \& Postma, D. (2005). Geochemistry, Groundwater and Pollution, Second Edition. Taylor \& Francis. doi:https://doi.org/10.1016/j.gca.2018.04.00310.1201/9781439833544.ch6.

Appelo, C. A. J., Van Loon, L. R., \& Wersin, P. (2010). Multicomponent diffusion of a suite of tracers (HTO, Cl, Br, I, Na, Sr, Cs) in a single sample of Opalinus Clay. Geochimica et Cosmochimica Acta, 74, 1201-1219. doi:https://doi.org/10.1016/j.gca.2018.04.00310.1016/j.gca.2009.11.013.

Appelo, C. A. J., \& Wersin, P. (2007). Multicomponent diffusion modeling in clay systems with application to the diffusion of tritium, iodide, and sodium in Opalinus Clay. Environmental Science 8 Technology, 41, 5002-5007. doi:https://doi.org/10.1016/j.gca.2018.04.00310.1021/es0629256.

Birgersson, M. (2017). A general framework for ion equilibrium calculations in compacted bentonite. Geochimica et Cosmochimica Acta, 200, 186-200.

Birgersson, M., \& Karnland, O. (2009). Ion equilibrium between montmorillonite interlayer space and an external solution - Consequences for diffusional transport. Geochimica et Cosmochimica Acta, 73, 1908-1923. doi:https://doi.org/10.1016/j.gca.2018.04.00310.1016/j.gca.2008.11.027.

Bourg, I. C., Beckingham, L. E., \& DePaolo, D. J. (2015). The nanoscale basis of $\mathrm{CO}_{2}$ trapping for geologic storage. Environmental Science \& Technology, 49, $10265-10284$. doi:https://doi.org/10.1016/j.gca.2018.04.00310.1021/acs.est.5b03003.

Bourg, I. C., Lee, S. S., Fenter, P., \& Tournassat, C. (2017). Stern layer structure and energetics at mica-water interfaces. Journal of Physical Chemistry C, 121, 9402-9412. doi:https://doi.org/10.1016/j.gca.2018.04.00310.1021/acs.jpcc.7b01828.

Bradbury, M. H., \& Baeyens, B. (1998). A physicochemical characterisation and geochemical modelling approach for determining porewater chemistries in argillaceous rocks. Geochimica et Cosmochimica Acta, 62, 783-795. doi:https://doi.org/10.1016/j.gca.2018.04.00310.1016/S0016-7037(97)00387-6.

Cussler, E. (1984). Diffusion - Mass Transfer in Fluid Systems. Cambridge University Press.

Delgado, A. V., Gonzalez-Caballero, F., Hunter, R. J., Koopal, L. K., \& Lyklema, J. (2005). Measurement and interpretation of electrokinetic phenomena. Pure and Applied Chemistry, 77, 1753-1805.

Descostes, M., Blin, V., Bazer-Bachi, F., Meier, P., Grenut, B., Radwan, J., Schlegel, M. L., Buschaert, S., Coelho, D., \& Tevissen, E. (2008). Diffusion of anionic species in Callovo-Oxfordian argillites and Oxfordian limestones (Meuse/Haute-Marne, France). Applied Geochemistry, 23, 655-677. doi:https://doi.org/10.1016/j.gca.2018.04.00310.1016/j.apgeochem.2007.11.003.

Donnan, F. G. (1911). Theorie der Membrangleichgewichte und Membranpotentiale bei Vorhandensein von nicht dialysierenden Elektrolyten. Ein Beitrag zur physikalisch-chemischen Physiologie (Theory of 
membrane equilibria and membrane potentials in the presence of non-dialysing electrolytes. A contribution to physical-chemical physiology). Zeitschrift für Elektrochemie und Angewandte Physikalische Chemie, 17, 572-581.

Flury, M., \& Gimmi, T. (2002). Solute Diffusion. In J. Dane, \& G. Topp (Eds.), Methods of Soil Analysis, Part 4-Physical Methods chapter 6.2. (pp. 1323-1351). Madison, Wisconsin, USA: Soil Science Society of America Inc.

Gimmi, T., \& Kosakowski, G. (2011). How mobile are sorbed cations in clays and clay rocks? Environmental Science \& Technology, 45, 1443-1449. doi:https://doi.org/10.1016/j.gca.2018.04.00310.1021/es1027794.

Gimmi, T., Leupin, O. X., Eikenberg, J., Glaus, M. A., Van Loon, L. R., Waber, H. N., Wersin, P., Wang, H. A. O., Grolimund, D., Borca, C. N., Dewonck, S., \& Wittebroodt, C. (2014). Anisotropic diffusion at the field scale in a 4-year multi-tracer diffusion and retention experiment - I: Insights from the experimental data. Geochimica et Cosmochimica Acta, 125, 373-393. URL: http://www.sciencedirect.com/science/article/pii/S001670371300570X. doi:https://doi.org/10.1016/j.gca.2018.04.003dx.doi.org/10.1016/j.gca.2013.10.014.

Glaus, M. A., Birgersson, M., Karnland, O., \& Van Loon, L. R. (2013). Seeming steady-state uphill diffusion of Na-22(+) in compacted montmorillonite. Environmental Science \& Technology, 47, 11522-11527. doi:https://doi.org/10.1016/j.gca.2018.04.00310.1021/es401968c.

Gonçalvès, J., Rousseau-Gueutin, P., \& Revil, A. (2007). Introducing interacting diffuse layers in TLM calculations: A reappraisal of the influence of the pore size on the swelling pressure and the osmotic efficiency of compacted bentonites. Journal of Colloid and Interface Science, 316, 92-99. doi:https://doi.org/10.1016/j.gca.2018.04.00310.1016/j.jcis.2007.07.023.

Hedström, M., \& Karnland, O. (2012). Donnan equilibrium in Na-montmorillonite from a molecular dynamics perspective. Geochimica et Cosmochimica Acta, 77, $266-274$. doi:https://doi.org/10.1016/j.gca.2018.04.00310.1016/j.gca.2011.11.007.

Jenni, A., Gimmi, T., Alt-Epping, P., Mäder, U., \& Cloet, V. (2017). Interaction of ordinary portland cement and opalinus clay: Dual porosity modelling compared to experimental data. Physics and Chemistry of the Earth, 99, 22-37. doi:https://doi.org/10.1016/j.gca.2018.04.00310.1016/j.pce.2017.01.004

Jougnot, D., Revil, A., \& Leroy, P. (2009). Diffusion of ionic tracers in the callovo-oxfordian clay-rock using the donnan equilibrium model and the formation factor. Geochimica et Cosmochimica Acta, 73, 2712-2726. doi:https://doi.org/10.1016/j.gca.2018.04.00310.1016/j.gca.2009.01.035.

Leroy, P., \& Revil, A. (2004). A triple-layer model of the surface electrochemical properties of clay minerals. Journal of Colloid and Interface Science, 270, 371-380. doi:https://doi.org/10.1016/j.gca.2018.04.00310.1016/j.jcis.2003.08.007.

Leroy, P., Revil, A., \& Coelho, D. (2006). Diffusion of ionic species in bentonite. Journal of Colloid and Interface Science, 296, 248-255.

Lichtner, P. (2007). FLOTRAN User's Manual: Two-phase nonisothermal coupled thermal-hydrologicchemical (THC) reactive flow and transport code, Version 2. Technical Report Los Alamos National Laboratory, Los Alamos, New Mexico.

Meyer, K. H., \& Sievers, I. F. (1936). The permeability of membranes I - The theory of ionic permeability. Helvetica Chimica Acta, 19, 649-664. doi:https://doi.org/10.1016/j.gca.2018.04.00310.1002/hlca.19360190199.

Mitchell, J. K. (1993). Fundamentals of Soil Behavior. (2nd ed.). Wiley.

Muniruzzaman, M., \& Rolle, M. (2016). Modeling multicomponent ionic transport in groundwater with IPhreeqc coupling: Electrostatic interactions and geochemical reactions in homogeneous and heterogeneous domains. Advances In Water Resources, 98, 1-15. doi:https://doi.org/10.1016/j.gca.2018.04.00310.1016/j.advwatres.2016.10.013.

Muurinen, A., Penttilä-Hiltunen, P., \& Uusheimo, K. (1989). Diffusion of chloride and uranium in compacted sodium bentonite. Materials Research Society Symposium Proceedings, 127, 743-748.

Nardi, A., Idiart, A., Trinchero, P., de Vries, L. M., \& Molinero, J. (2014). Interface COMSOL-PHREEQC (iCP), an efficient numerical framework for the solution of coupled multiphysics and geochemistry. Computers \& Geosciences, 69, 10-21. doi:https://doi.org/10.1016/j.gca.2018.04.00310.1016/j.cageo.2014.04.011.

Neuzil, C. E., \& Person, M. (2017). Reexamining ultrafiltration and solute transport in groundwater. Water Resources Research, 53, 4922-4941. doi:https://doi.org/10.1016/j.gca.2018.04.00310.1002/2017WR020492.

Parkhurst, D. L., \& Wissmeier, L. (2015). PhreeqcRM: A reaction module for transport simulators based on the geochemical model PHREEQC. Advances In Water Resources, 83, 176-189. doi:https://doi.org/10.1016/j.gca.2018.04.00310.1016/j.advwatres.2015.06.001. 
Sørensen, T. S., \& Compañ, V. (1996). Salt flux and electromotive force in concentration cells with asymmetric ion exchange membranes and ideal 2:1 electrolytes. Journal of Physical Chemistry, 100, 15261-15273. doi:https://doi.org/10.1016/j.gca.2018.04.00310.1021/jp9609895.

Steefel, C. I. (2008). Geochemical Kinetics and Transport. Kinetics of Water-Rock Interaction chapter 11. (pp. 545-589). Springer.

Steefel, C. I., Appelo, C. A. J., Arora, B., Jacques, D., Kalbacher, T., Kolditz, O., Lagneau, V., Lichtner, P. C., Mayer, K. U., Meeussen, J. C. L., Molins, S., Moulton, D., Shao, H., Šimůnek, J., Spycher, N., Yabusaki, S. B., \& Yeh, G. T. (2015). Reactive transport codes for subsurface environmental simulation. Computational Geosciences, 19, 445-478. URL: http://dx.doi.org/10.1007/s10596-014-9443-x. doi:https://doi.org/10.1016/j.gca.2018.04.00310.1007/s10596-014-9443-x.

Tinnacher, R. M., Holmboe, M., Tournassat, C., Bourg, I. C., \& Davis, J. A. (2016). Ion adsorption and diffusion in smectite: Molecular, pore, and continuum scale views. Geochimica et Cosmochimica Acta, 177, 130-149. doi:https://doi.org/10.1016/j.gca.2018.04.00310.1016/j.gca.2015.12.010.

Tournassat, C., \& Appelo, C. A. J. (2011). Modelling approaches for anion-exclusion in compacted Na-bentonite. Geochimica et Cosmochimica Acta, 75, 3698-3710.

Tournassat, C., Bourg, I. C., Holmboe, M., Sposito, G., \& Steefel, C. I. (2016). Molecular dynamics simulations of anion exclusion in clay interlayer nanopores. Clays and Clay Minerals, 64, 374-388. doi:https://doi.org/10.1016/j.gca.2018.04.00310.1346/CCMN.2016.0640403.

Tournassat, C., \& Steefel, C. I. (2015). Ionic transport in nano-porous clays with consideration of electrostatic effects. Reviews in Mineralogy \& Geochemistry, Pore-scale Geochemical Processes, 80, 287-329. doi:https://doi.org/10.1016/j.gca.2018.04.00310.2138/rmg.2015.80.09.

Van Loon, L. R., Glaus, M. A., \& Müller, W. (2007). Anion exclusion effects in compacted bentonites: Towards a better understanding of anion diffusion. Applied Geochemistry, 22, 2536-2552.

Van Loon, L. R., Wersin, P., Soler, J. M., Eikenberg, J., Gimmi, T., Hernan, P., Dewonck, S., \& Savoye, S. (2004). In-situ diffusion of HTO, Na-22(+), Cs + and I- in Opalinus Clay at the Mont Terri underground rock laboratory. Radiochimica Acta, 92, 757-763.

Wersin, P., Soler, J. M., Van Loon, L., Eikenberg, J., Baeyens, B., Grolimund, D., Gimmi, T., \& Dewonck, S. (2008). Diffusion of HTO, Br-, I-, Cs+, Sr-85(2+) and Co-60(2+) in a clay formation: Results and modelling from an in situ experiment in Opalinus Clay. Applied Geochemistry, 23, 678-691.

Wigger, C., \& Van Loon, L. R. (2017). Importance of interlayer equivalent pores for anion diffusion in clay-rich sedimentary rocks. Environmental Science \& Technology, 51, 1998-2006. doi:https://doi.org/10.1016/j.gca.2018.04.00310.1021/acs.est.6b03781.

Wissmeier, L., \& Barry, D. A. (2011). Simulation tool for variably saturated flow with comprehensive geochemical reactions in two- and three-dimensional domains. Environmental Modelling $\mathscr{G}$ Software, 26, 210-218. doi:https://doi.org/10.1016/j.gca.2018.04.00310.1016/j.envsoft.2010.07.005. 